\title{
GESTIÓN DE COSTOS DE DESECHOS Y DESPERDICIOS EN LAS MYPES DE LA CONFECCIÓN
}

\author{
LEFTOVER MANAGEMENT COSTS AND WASTE IN CLOTHING MYPES \\ Elsie Bonilla Pastor* \\ Unidad de Posgrado de la Facultad de Ciencias Contables \\ Universidad Nacional Mayor de San Marcos-UNMSM / Lima-Perú \\ [Recepción: Febrero de 2014 / Conformidad: Febrero de 2014]
}

\section{RESUMEN}

Las MYPE's del Perú representan el 99.3\% del total de empresas con iniciativas económicas y generan el $75 \%$ del empleo. Sin embargo, a diferencia de lo que ocurre en países desarrollados como USA y Japón, su nivel de competitividad es bajo y su condición financiera es frágil, siendo su participación en las exportaciones menor que $1 \%$. La inadecuada gestión de la calidad de sus procesos, genera desechos y desperdicios, que incrementan los costos, reduciendo su productividad y rentabilidad. La investigación realizada sobre una muestra de 27 MYPE's de la confección textil de prendas ubicadas en Lima y Callao, concluye que existe una correlación inversa y significativa entre la calificación de la gestión de la calidad y los costos por desechos y desperdicios, hallándose que por cada $1 \%$ de calificación adicional a la gestión de la calidad se reducen los costos de producción, en $0.08 \%$; también se identifican los factores de la gestión que tienen mayor impacto.

\section{Palabras clave:}

Sistema de gestión de calidad; costo de desechos y desperdicios; MYPE's de la confección textil de prendas; costo de producción; Norma ISO 9001: 2008.

\begin{abstract}
Peruvian MYPEs represent $99.3 \%$ of all businesses with economic initiatives and generate $75 \%$ of employment. However, opposite of what happens in developed countries like USA and Japan, their competitiveness is low and the financial condition is fragile, moreover their export share of less than $1 \%$. Inadequate quality management of their processes generates waste and scrap, which increase costs, reducing their productivity and profitability. Research conducted on a sample of 27 textile MYPEs-garments manufacturers- located in Lima and Callao, concluded that there is an inverse and significant correlation between the rating of the quality management and the costs of waste and scrap; and found that for every $1 \%$ of additional quality management qualification, production costs are reduced at $0.08 \%$; we also identified the greatest impact management factors.
\end{abstract}

\section{Keywords:}

Quality management system; cost of waste and scrap; MYPE's textile garment manufacturers; production cost; ISO 9001: 2008.

* Doctorante en Ciencias Contables y Empresariales - UNMSM. Email: elsiebonilla@gmail.com 


\section{INTRODUCCIÓN}

El sector textil de la confección es el segundo más importante del sector manufacturero en términos del número de empresas. En dicho sector, solo el 0.4\% son grandes empresas y el $94.7 \%$ son microempresas; según la SUNAT, se estima que dicho sector está representado por 20,755 establecimientos formales, estando el 51\% ubicado en Lima y Callao, y la PEA relacionada se estima en 139,456 habitantes (Perú. Ministerio de la Producción, 2011). Un informe presentado en el año 2005 por el Instituto Español de Comercio Exterior (ICEX, 2005), luego de realizar un diagnóstico al sector textil de la confección, plantea que si bien los tratados de libre comercio firmados con los países del norte representan una oportunidad para las exportaciones textiles, en el Perú existe un sector fragmentado con presencia de PYMES poco competitivas y de frágil condición financiera, lo cual conlleva a que las exportaciones se concentren en pocas empresas, generalmente de gran tamaño. El nivel de competitividad de tales empresas se ve afectada por el nivel de calidad de los productos y servicios correspondientes, los niveles de precio y la baja inversión tecnológica.

La presente investigación tiene como principal motivación, contribuir con nuevo conocimiento para el mejoramiento de la calidad de los procesos y productos de las MYPE's peruanas de la confección textil, y en consecuencia, con el incremento de su productividad. Quienes conducen las pequeñas y microempresas no tienen claro la importancia de gestionar adecuadamente la calidad de sus procesos productivos, desde la etapa de diseño hasta el pro- ducto terminado, perjudicando el cumplimiento de los requisitos de calidad y generando altos niveles de desechos y desperdicios, cuyo valor económico es ignorado; así mismo, la falta de registros y documentación de la gestión impide la búsqueda racional de las causas raíz asociadas y no facilita la mejora continua e innovación de los procesos.

El “Padre de la calidad”, Deming, E (1986) manifiesta que: "el fracaso de la gestión para planificar el futuro y predecir los problemas ha traído como consecuencia un despilfarro de mano de obra, materiales y de tiempo-máquina, todo lo cual incrementa el costo del fabricante y el precio que debe pagar el comprador"; así mismo, explica que la causa fundamental de la enfermedad de la industria americana y el consecuente desempleo es el fracaso de la dirección, que no dirige, pues aquel que no vende no puede comprar. La dirección, manifiesta, debe comprender el diseño del producto y del servicio, la adquisición de materiales, los problemas de producción, el control del proceso, y las barreras que privan al trabajador del derecho de estar orgullosos de su trabajo. Se dice que cuando el señor Deming preguntó a 22 operarios de una fábrica: ¿Por qué sucede que al mejorar la calidad aumenta la productividad?, la respuesta unánime fue...” No hay desperdicios”. Al mejorar la calidad, se transfieren las horas hombres y las horas máquinas malgastadas en la fabricación de producto bueno y a dar un servicio mejor. El resultado es una reacción en cadena: se reduce los costos, se es más competitivo, la gente está más contenta en su trabajo, hay trabajo y más trabajo. Un esquema que grafica la ruta de lo señalado por Deming, se muestra a continuación: 
Figura $\mathbf{N}^{\circ}$ 01: Esquema de la mejora de la calidad

\begin{tabular}{|c|}
\hline Mejor calidad \\
\hline$\downarrow$ \\
\hline $\begin{array}{c}\text { Decrecen los costes porque hay menos reprocesos, menos } \\
\text { equivocaciones, menos retrasos; se utilizan mejor el tiempo - } \\
\text { máquina y los materiales }\end{array}$ \\
\hline$\downarrow$ \\
\hline Mejora la productividad \\
\hline$\downarrow$ \\
\hline Se conquista un mayor mercado con la calidad y menor precio \\
\hline$\downarrow$ \\
\hline Se permanece en el negocio \\
\hline$\downarrow$ \\
\hline Hay más y más trabajo \\
\hline
\end{tabular}

Fuente: Out of the Crisis. Edward Deming (1986)

Cuadro $\mathbf{N}^{\circ}$ 01: Mejoras luego de implementar un Sistema de Gestión de la Calidad

\begin{tabular}{|l|c|c|}
\hline \multicolumn{1}{|c|}{ Rubro } & $\begin{array}{c}\text { Antes de la mejora } \\
(\mathbf{1 1 \%} \text { defectuosos) }\end{array}$ & $\begin{array}{c}\text { Después de la mejora } \\
\text { (5\% defectuosos) }\end{array}$ \\
\hline Coste total & 100 & 100 \\
\hline $\begin{array}{l}\text { Desembolso por hacer unidades } \\
\text { buenas }\end{array}$ & 89 & 95 \\
\hline $\begin{array}{l}\text { Desembolso por hacer unidades } \\
\text { defectuosas. }\end{array}$ & 11 & 5 \\
\hline Número de unidades defectuosas & 11 & 5 \\
\hline
\end{tabular}

Fuente: Out of the Crisis. Edward Deming (1986)

Beneficios:

- Mejor calidad.

- La capacidad se incrementa en un 6 por 100.

- Coste inferior por unidad de producto bueno. Más beneficios.

- La producción de artículos buenos se incrementa en $6 \%$.

La baja calidad significa costes elevados y cuando un director comprende la magnitud del problema; es decir, pagar dinero por hacer defectos, así como, para corregirlos, buscará maneras de mejorar los procesos y de ayudar a las personas en la línea para que entiendan mejor cómo hacer su trabajo. El coste del reproceso es solo una parte del coste de la mala calidad.
La mala calidad disminuye la productividad a lo largo de toda la línea de la actividad de producción, y parte del producto defectuoso llega a las manos del cliente, quien a la vez, lo transmite a sus amigos. El efecto multiplicador de un cliente disgustado es una de esas cifras desconocidas e incognoscibles, y lo mismo ocurre con el efecto multiplicador de un cliente satisfecho, que atrae más clientes.

A. V. Feigenbaum estimó que entre el 15\% - 40\% de los costes de fabricación de casi todos los productos americanos que se vendían en su época, se debían al desperdicio incluido en el desperdicio de esfuerzo humano, desperdicio de tiempo-máquina y uso no productivo de las cargas incluidas. 
Lefcovich (2009) opina que el sistema Kaizen de mejora continua tiene como uno de sus pilares fundamentales la lucha continua en la eliminación de desperdicios y despilfarros (mudas en japonés). Una lucha implacable y sin respiro en la necesidad de eliminar los factores generadores de improductividades, altos costos, largos ciclos, costosas y largas esperas, desaprovechamiento de recursos, pérdida de clientes, y defectos de calidad; todo lo cual origina la pérdida de participación en el mercado con caída en la rentabilidad y en los niveles de satisfacción de los consumidores. Una empresa que no controla sus desperdicios, que no tiene noción de ellas, y que por tanto no adopta medidas para prevenirlas o eliminar sus causas gestará productos y servicios de mala calidad, con altos costos y malos servicios, o sea bienes con un bajo valor para los clientes, por lo que ellos no estarán dispuesto a su adquisición o sólo lo harán a un muy bajo precio.

Uno de los sistemas de gestión de la calidad más utilizados a nivel mundial y nacional es el Modelo de Gestión ISO 9001:2008, cuyos requisitos se muestran en la siguiente figura:

Figura $N^{\circ}$ 02: Requisitos de la Norma ISO 9001
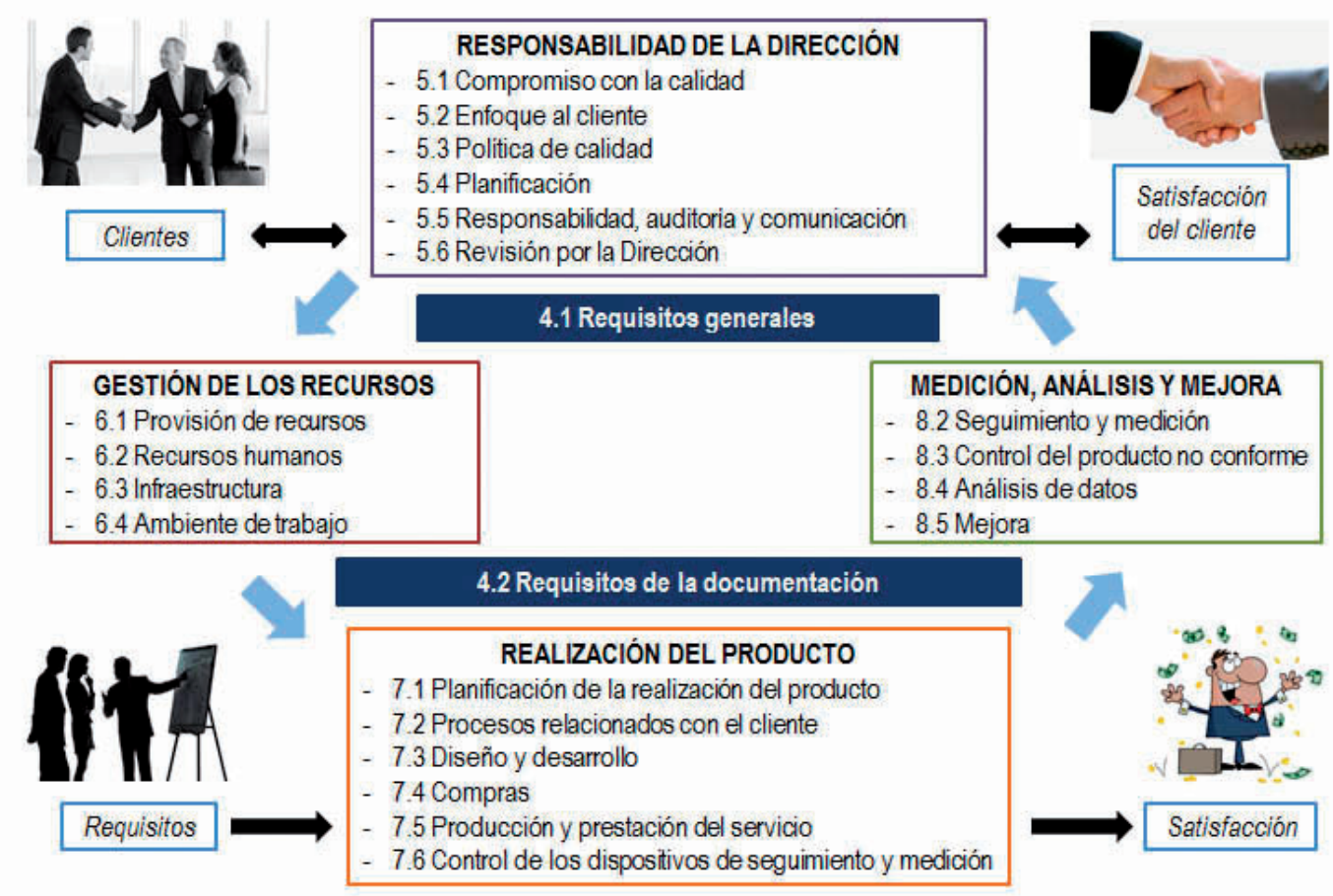

Fuente: Norma ISO 9001:2008

El objetivo general de la investigación fue demostrar que existe una relación entre la valoración de la gestión de la calidad en la MYPEs de la confección textil de Lima y Callao, que elaboran prendas con tejido de punto o tejido plano, y los niveles de costos generados por desechos y desperdicios. Los objetivos específicos se orientaban a identificar los factores o aspectos de la gestión de calidad que mejor se correlacionan con los niveles de costos por desechos y desperdicios, los mismos que según la subcuenta 222 del Plan Contable General del Perú, se refieren a materiales desechados por presentar defectos o que resultan no utilizables en el proceso de transformación.

El estudio halló con la restricción de tener que cuantificar objetivamente las mermas generadas en las diversas etapas del proceso productivo en vista que carecían de información registrada al respecto. 


\section{METODOLOGÍA}

El estudio se realizó de manera transversal, descriptiva y correlacional sobre una muestra representativa de 27 MYPE's formales de la confección textil de prendas en tejido de punto y tejido plano; la información colectada corresponde al año 2012. El análisis se ha enfocado a la gestión de los procesos de producción, conformado por los siguientes sub-procesos: corte y habilitado, confección de piezas, armado y acabado.

La metodología utilizada incluyó:

a) Entrevistas con los conductores de las empresas a fin de explicar los objetivos de la investigación y los instrumentos a utilizar para la colecta de información.

b) Aplicación de un primer cuestionario al conductor o jefe de producción de la empresa. El instrumento permitió valorizar del Sistema de Gestión de la Calidad (SGC) de cada unidad y su estructura se ha basado en modelo de gestión ISO 9001:2008.

c) Aplicación del segundo cuestionario, para cuantificar los desechos y desperdicios generados en cada etapa del proceso productivo y expresar su costo como un porcentaje del costo de producción. La falta de registros creó la necesidad de validar las respuestas, con observaciones directas al proceso productivo.

d) Entrevista con expertos (Técnica Delphi) para validar algunas conclusiones y brindar mayor soporte al análisis.

El procesamiento de la información y comprobación de las hipótesis planteadas utilizaron los software MINITAB y SPSS, también se aplicaron pruebas de normalidad, correlación, regresión y pruebas estadísticas de significancia de Pearson, Spearman, Wilcoxon y la prueba paramétrica de T student.

\section{RESULTADOS DE LA INVESTIGACIÓN}

A continuación presentamos los principales resultados obtenidos:

1. Valoración de la Gestión de la Calidad de las MYPE's de la confección textil

Figura $\mathrm{N}^{\circ}$ 03: Valoración de la implementación del SGC en cada MYPE

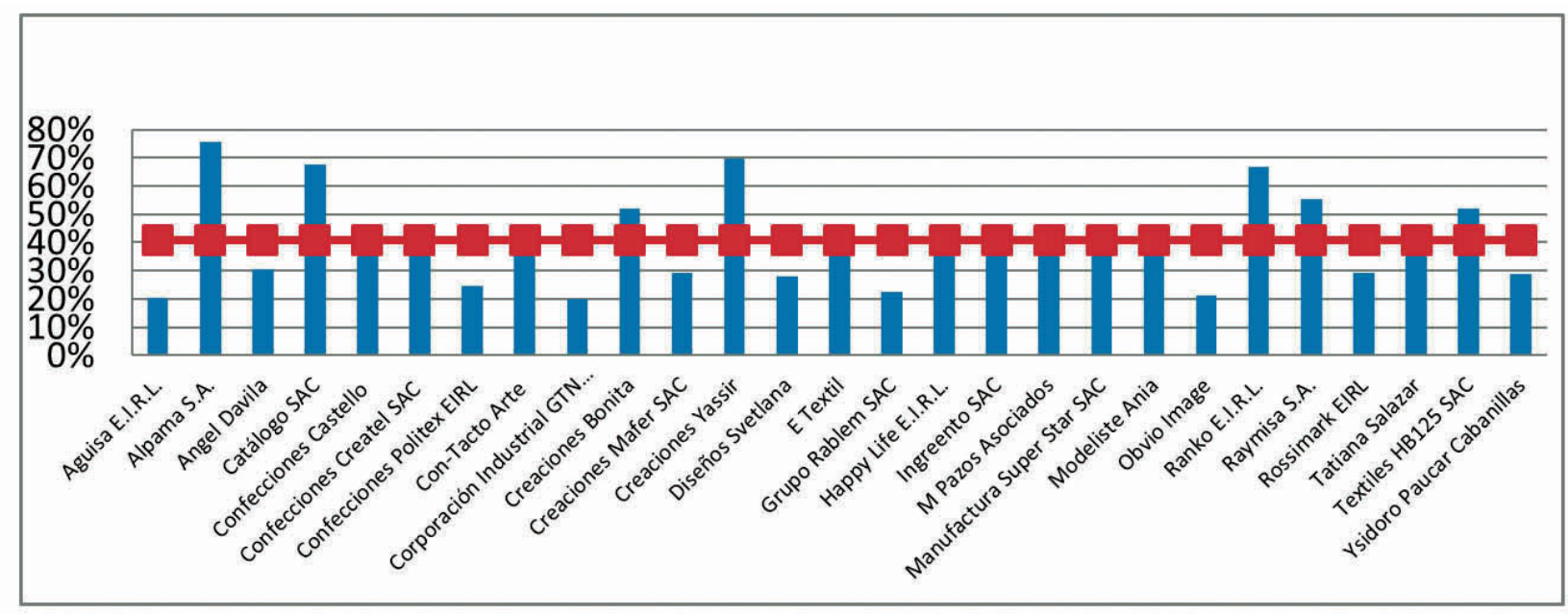

Fuente: Cuestionarios aplicados a las empresas. Elaboración propia.

Con relación a la valorización del SGC, la calificación se halla en el rango de $20 \%$ a $75 \%$, siendo el promedio global de casi $41 \%$. Se pudo observar que $35 \%$ de las empresas obtuvieron una valoración por debajo de $30 \%$, otro $35 \%$ estuvo entre 30 y $50 \%$, y el $30 \%$ restante logró una calificación entre 50 y $78 \%$.
El cuestionario utilizado se compone de los siguientes factores:

1. Documentación del Sistema de Gestión de la Calidad (X1).

2. Responsabilidad de la Dirección (X2). 
3. Gestión del Recurso (X3).

4. Gestión de la realización de la prenda textil (X4).

5. Medición, análisis y mejora (X5).

Las valoraciones promedio para cada factor se muestran en la figura $\mathrm{N}^{\circ} 04$; se observa que el área con mayor calificación (51.41\%) es el de "Responsabilidad de la Dirección”, dicho aspecto califica el compromiso de los conductores de las MYPE's, con el desarrollo e implementación del SGC. El área de menor calificación es el de "Medición, análisis y mejora" (30.96\%), aspecto que se refiere al esfuerzo de la organización para medir, registrar y analizar las incidencias en los procesos productivos que afecten la calidad, y el esfuerzo para eliminar las causas raíz y en consecuencia, promover la mejora continua.

Figura $\mathrm{N}^{\circ} 04$ Valoración promedio de los factores del SGC

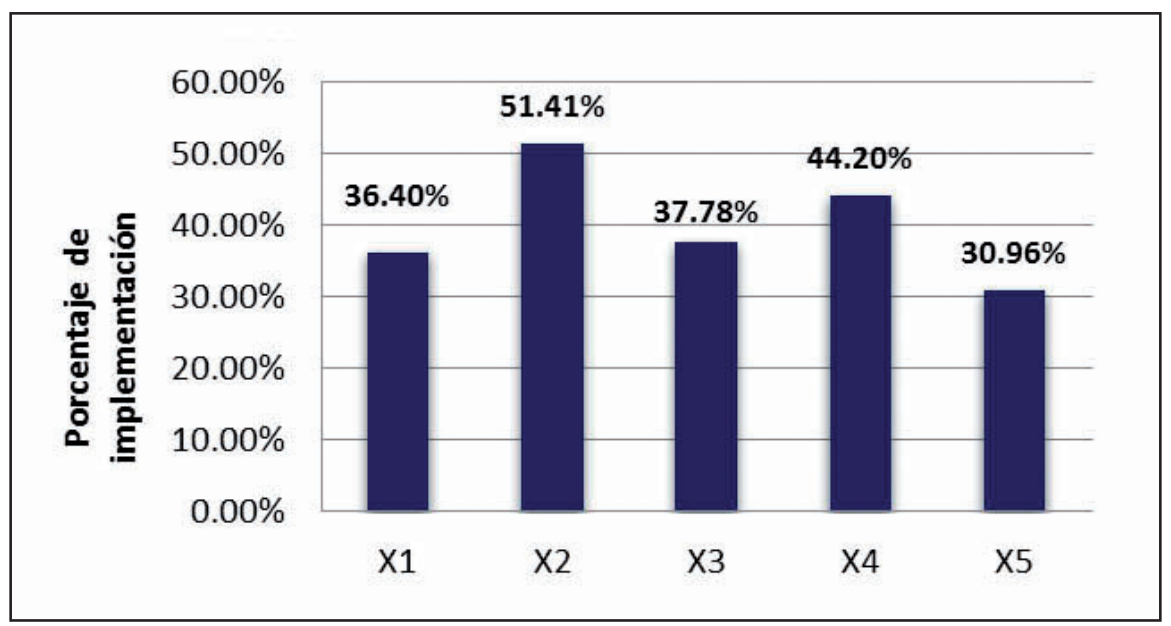

Fuente: Cuestionarios aplicados a las empresas. Elaboración propia.

Se procede a mostrar el detalle de la calificación de cada factor o aspecto evaluado, el mismo que se compone de diversas preguntas que fueron calificadas con la escala de Likert; así mismo se muestra el valor promedio alcanzado por el aspecto.

Figura $\mathbf{N}^{\circ}$ 05: Puntaje promedio del factor: Documentación del SGC

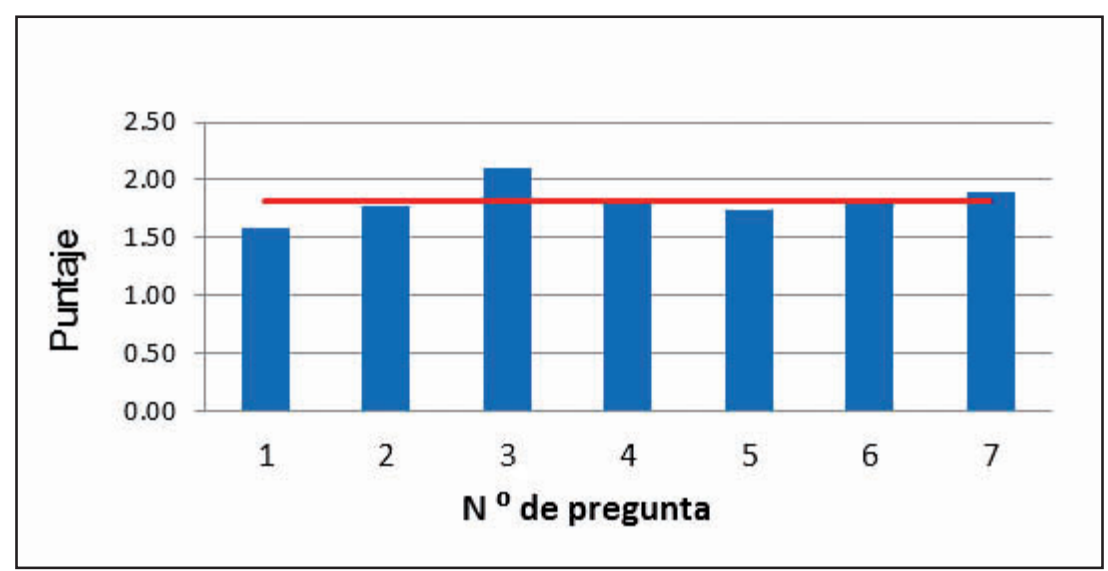

Fuente: Cuestionarios aplicados a las empresas. Elaboración propia.

El puntaje máximo corresponde a la pregunta 3 que se refiere a la existencia del organigrama y del Manual de Funciones y Responsabilidades; el menor puntaje lo alcanza el ítem 1 que se refiere a la existencia de un Manual de Calidad. 
Figura $\mathbf{N}^{\circ}$ 06: Puntaje promedio del factor: Responsabilidad de la Dirección

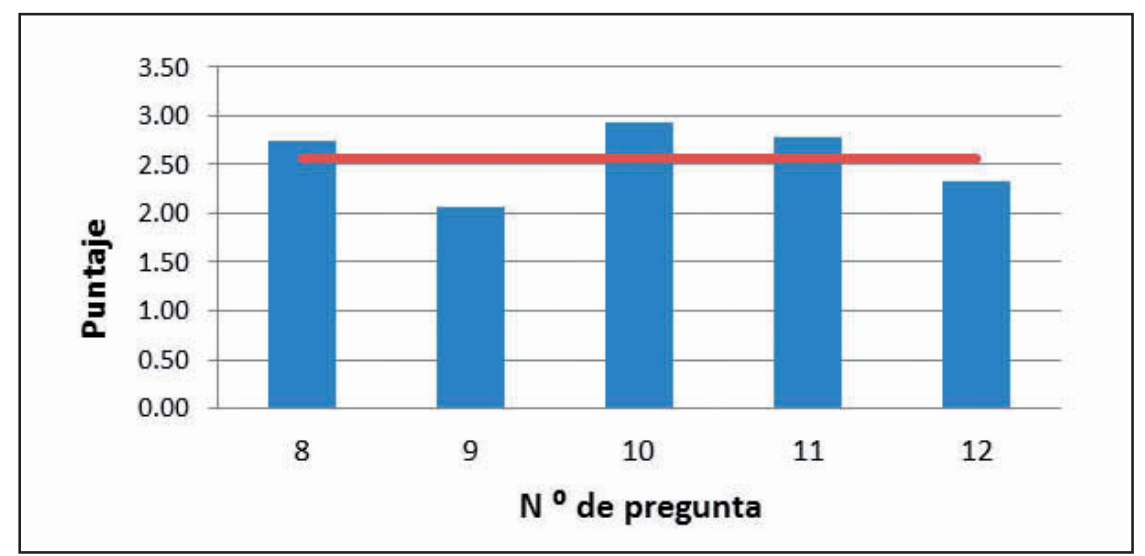

Fuente: Cuestionarios aplicados a las empresas. Elaboración propia.

La pregunta de más baja valoración (9) es: "La Dirección tiene un Plan de Calidad para lograr los objetivos de calidad", y la de más alta valoración (10): "La Dirección facilita la disponibilidad de recursos para el buen desempeño de los procesos".

Figura $\mathbf{N}^{\circ}$ 07: Puntaje promedio del factor: Gestión de la realización de la prenda textil

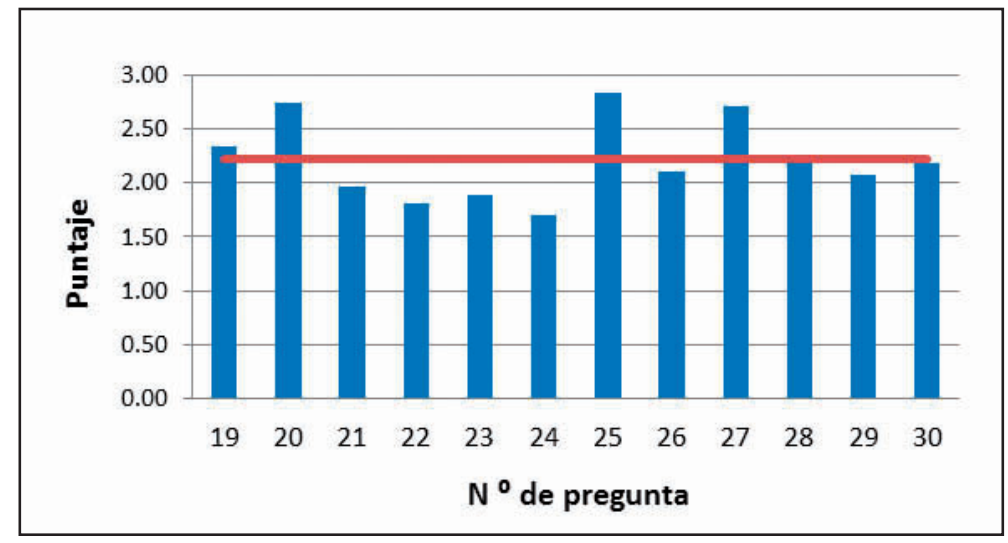

Fuente: Cuestionarios aplicados a las empresas. Elaboración propia.

Las preguntas de más baja valoración fueron:

- La empresa ha elaborado un Plan de Control de Calidad.

- La empresa mantiene los registros que demuestran la revisión del cumplimiento de los requisitos de calidad en materiales y productos.

- La empresa aplica sistemáticamente encuestas para conocer el nivel de satisfacción de los clientes.
De otro lado, se pudo observar que entre las preguntas de mayor valoración se encuentran:

- El uso de documentos para la compra de materiales, donde básicamente se describe el producto, la cantidad y el precio.

- El cuidado del proceso de diseño y desarrollo de productos.

- La observancia de las fichas técnicas entregadas por los clientes. 
Figura $\mathbf{N}^{\circ}$ 08: Puntaje promedio del factor: Medición, análisis y mejora

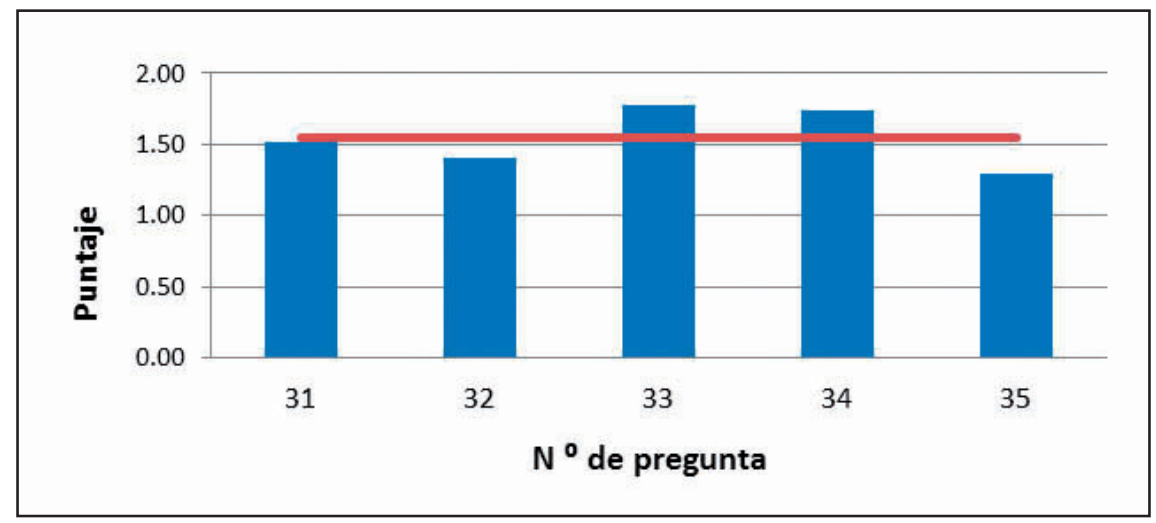

Fuente: Cuestionarios aplicados a las empresas. Elaboración propia.

Con relación al rubro de Medición, análisis y mejora, este fue el aspecto con el menor promedio de implementación; todos los ítems evaluados logran una calificación menor de 2 puntos, siendo el promedio de 1.5. Las PYME's de la confección textil de Lima y Callao no aplican métodos y registros para la identificación, seguimiento y medición de los procesos, lo cual impide identificar el nivel de conformidad con los requisitos del producto, ni emprende acciones correctivas y preventivas para eliminar las causas de la no conformidad.

\section{Resultados de la aplicación del Cuestionario 2: Contabilización de desechos y desperdicios}

La medición de los porcentajes promedio de desechos y desperdicios en cada etapa del proceso, se muestran en la figura $\mathrm{N}^{\circ}$ 9; se aprecia que la tasa más alta lo tiene al proceso de corte y habilitado (9.93\%) $y$ el menor es el generado en la etapa de acabado y planchado (1.89\%). Posteriormente, se calcula el costo representado por los desechos y desperdicios, y se le relaciona con el costo de producción correspondiente.

Figura $\mathbf{N}^{\circ}$ 09: Porcentaje promedio de desperdicios y desechos en las MYPE's

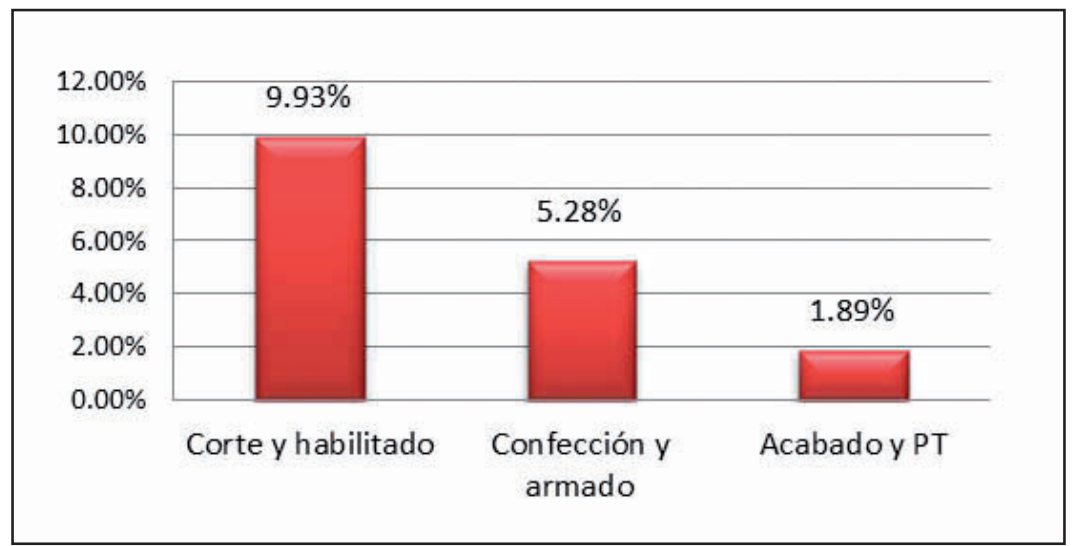

Fuente: Cuestionarios aplicados a las empresas. Elaboración propia. 
Figura $\mathbf{N}^{\circ}$ 10: Costos de desperdicios y desechos como porcentaje del costo

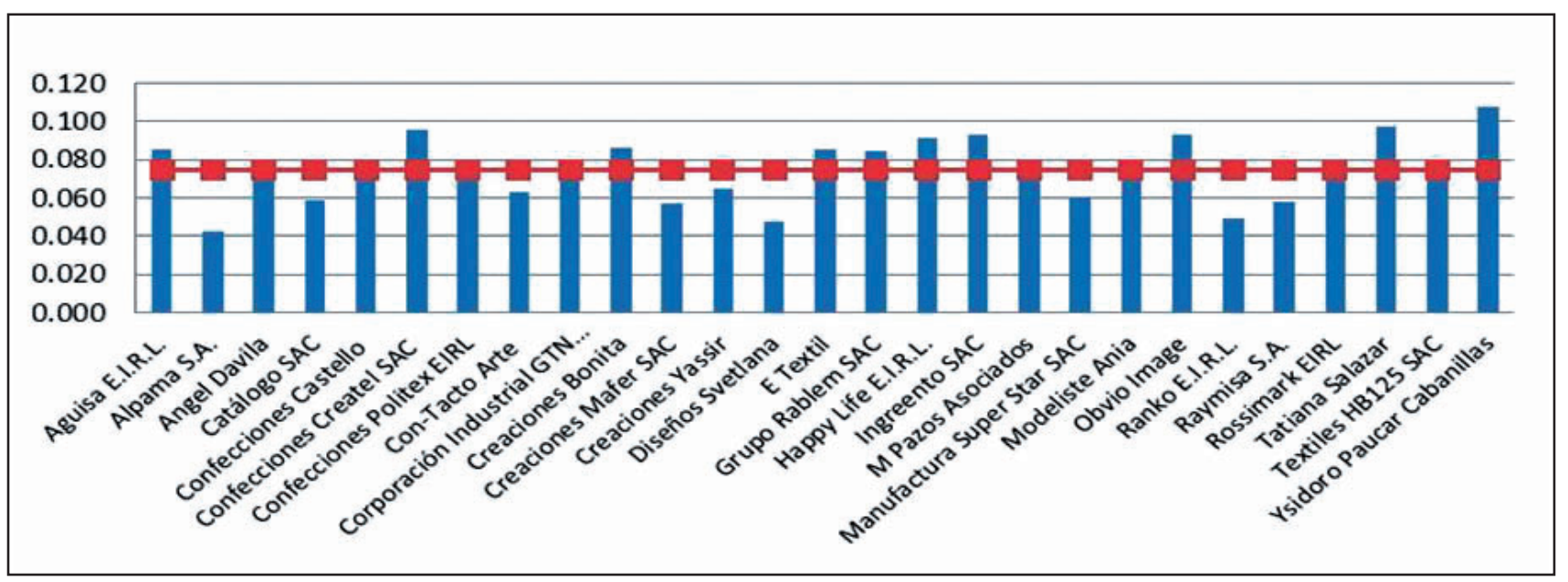

Fuente: Cuestionarios aplicados a las empresas. Elaboración propia.

Se puede observar que el indicador: costo de desechos y desperdicios / costo de producción, está comprendido entre $4.3 \%$ y $10.7 \%$; las diferencias con una media de $7.4 \%$.

\section{Análisis de la correlación entre la calificación del SGC y los costos de desechos y desperdicios}

La investigación evaluó la normalidad de las variables: "Valoración del SGC de las MYPE's de la confección textil" (X) y la variable dependiente, "Costo de desecho y desperdicios, como porcentaje del costo de producción” (Y), para luego establecer la relación entre ambas. Utilizando la prueba de Pearson $y$ con un alto nivel de significancia se determina que la relación inversa queda expresada con la función lineal: $\mathrm{Y}=10.7-0.0803 \mathrm{X}$.

El coeficiente de determinación ( $\mathrm{r} 2$ ) es de 0.588 y coeficiente de correlación ( $\mathrm{r}$ ) de 0.7668 .

Por cada $1 \%$ de calificación adicional al SGC de la empresa se puede reducir en $0.08 \%$, el costo de producción.

A continuación se muestra dicha relación en forma gráfica:

Figura $\mathrm{N}^{\circ}$ 11: Costos de desperdicios y desechos como porcentaje del costo de producción

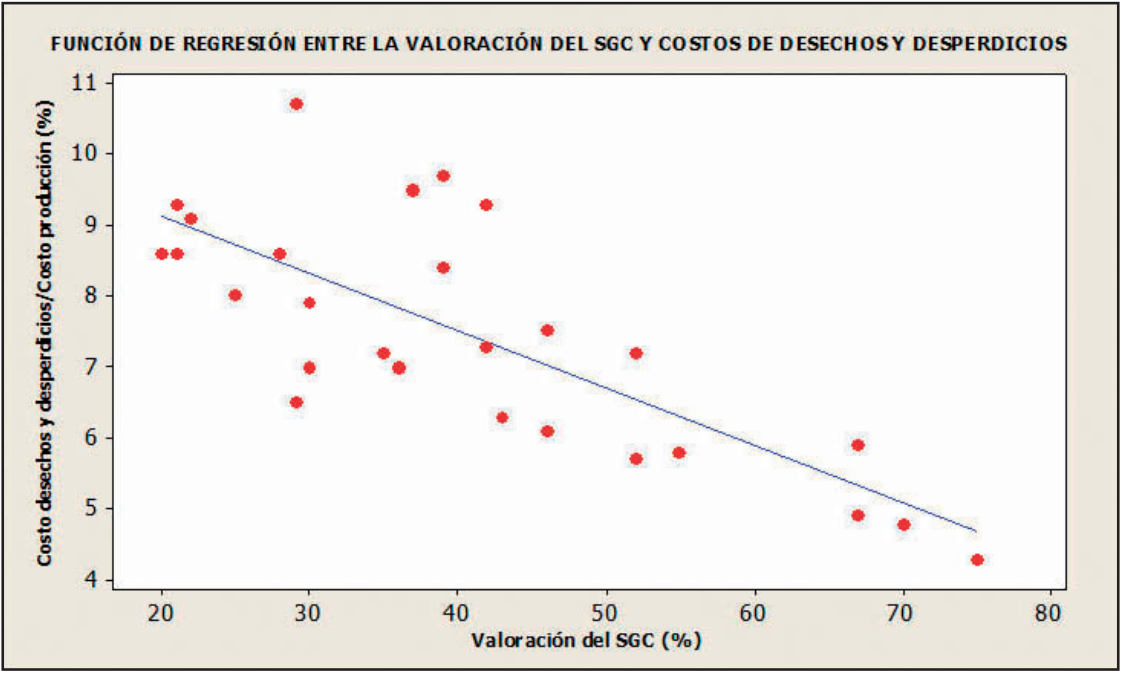

Fuente: Cuestionarios aplicados a las empresas. Elaboración propia. 


\section{Resultados de la reunión con los expertos del sector (Técnica DELPHI)}

La reunión convocó a 18 expertos MYPE's, provenientes del Ministerio de la Producción, Cámara de Comercio de Lima, COFIDE, SENATI, SNI, UNI, ITESSA, QualityLab, Molina Calidad total, entre otros; aquellos respondieron a un cuestionario con 11 preguntas, donde la de mayor puntuación (4.6 en la escala de Likert) fue: "La eficacia de la gestión de la calidad influye directamente en los niveles de desechos y desperdicios generados en la cadena productiva de las PYMES de la Confección textil"; así mismo, más del $50 \%$ opinó que los principales factores de la gestión que determinan la productividad y calidad en las MYPE's de la confección textil son: El compromiso y liderazgo de la dirección, y la competencia y experiencia del personal dedicado a la producción.

\section{CONCLUSIONES}

1. Existe una correlación inversa y fuerte entre la valoración (calificación) de la gestión de la calidad y los niveles de costos por desechos y desperdicios generados en las diferentes etapas del proceso productivo de las MYPES de la confección textil de Lima y Callao.

2. Los cinco factores del SGC (Sistema de Gestión de la Calidad) fueron encontrados como predictores estadísticamente significativos $(\mathrm{p}<0.05)$ del indicador: costo de desecho y desperdicio/costo de producción. Asimismo, se ha determinado que los factores que muestran un coeficiente de correlación "fuerte" son:

- Documentación del SGC.

- Gestión de la realización de la prenda textil.

3. El nivel de implementación de un SGC en las MYPEs de la confección textil de Lima y Callao alcanza un promedio de casi $41 \%$, de un rango de calificación entre $20 \%$ y $75 \%$.

4. El factor "Medición, análisis y mejora continua" muestra la más baja calificación (30\%) con relación a los otros factores del SGC, debido principalmente al perfil profesional de los conductores de las MYPE's, que no promueven una gestión formal, documentada y cuyas decisiones no se basan en resultados.

5. Existe una correlación directa entre el número de trabajadores y la valoración lograda por el SGC, lo cual podría justificarse por una mayor disponibilidad de recursos para capacitación, mejor nivel profesional de los conductores y mayor compromiso con el mercado.

6. La eficacia de la gestión de la capacitación influye directamente y de manera significativa en la valoración del SGC. En un $40 \%$ de las veces, la calificación del SGC queda explicado por la administración de la capacitación y de las competencias; un empleado capacitado con el objetivo de reforzar sus habilidades, tendrá una mayor cantidad de recursos técnicos y emocionales que le permitirán planificar, ejecutar, controlar y mejorar su trabajo.

\section{RECOMENDACIONES}

1. Utilizar la ecuación de regresión entre la valoración o calificación del SGC y el indicador de costos de desechos y desperdicios, para estimar los costos por desechos y desperdicios en el sector de la confección textil de prendas en las MYPE's peruanas, y como recursos de futuras investigaciones relacionadas.

2. Fortalecer los aspectos de gestión: "Documentación del SGC" y "Gestión de la realización de la prenda textil”. Un instrumento estratégico de refuerzo sería el Balanced Scorecard (Cuadro de Mando Integral), a fin de evaluar sistemáticamente los resultados de desempeño; su diseño puede ser parte de una investigación futura.

3. Que CODEMYPE (Consejo Nacional para el Desarrollo de la Micro y Pequeña Empresa) promueva la implementación de un modelo de gestión de la calidad como el propuesto por la presente investigación a fin de contribuir con la eficacia de la gestión y con ello, la mejora de su competitividad.

4. Las universidades, institutos técnicos como SENATI y diferentes gremios empresariales deben ofrecer cursos y talleres que permitan la 
capacitación de los conductores y empleados de las MYPE's de la confección textil, en la administración de la mejora continua de sus procesos y productos.

5. Se sugiere sensibilizar a las MYPE's de la confección textil sobre las ventajas de la implementación temprana del modelo de gestión, como el que se propone, para evitar que el negocio sea abortado por el mercado en su etapa inicial.

6. Las universidades y organizaciones que promueven el desarrollo y competitividad de las MYPE's en el país, deben diseñar un modelo de gestión del “Talento Humano", el cual además de desarrollar las competencias técnicas, permita elevar el nivel de inteligencia emocional de los conductores y trabajadores, contribuyendo con el clima laboral y calidad de vida en la sociedad, así como, con la eficacia y rentabilidad de dichas empresas.

\section{REFERENCIAS BIBLIOGRÁFICAS}

1. AlbERTO, A. (1994). La calidad y su costo. Buenos Aires: Addison Wesley Iberoamericana.

2. ALBERTO, A. (1995). Aplicación de ISO 9000 y cómo implementarlo. USA: Addison Wesley Iberoamericana .

3. BONILLA, E. (2010). Mejora continua de los procesos: Herramientas y técnicas (Primera ed.). Lima: Universidad de Lima - Fondo Editorial.

4. DEMING, W. E. (1986). Out of the Crisis: Quality, Productivity and Competitive Position. Cambridge University Press.

5. FLORES, A. A. (2004). Metodología de gestión para las micro, pequeñas y medianas empresas en Lima Metropolitana. Tesis para optar el Grado Académico de Doctor en Ciencias Contables y Empresariales, Lima.

6. IMILCE, M. (s.f.). MYPE competitiva, una MYPE con calidad. Guía para entender y evaluar un sistema de gestión de la calidad. Proyecto USAID/ PERU.
7. Instituto Español de Comercio Exterior - ICEX. (2005). Informes sectoriales. El sector textil y la confección en el Perú.

8. ISO 9001. (2012). Recuperado el 9 de setiembre de 2013, de DataBase Survey 2012:http://www. iso.org/iso/home/standards/certification/isosurvey.htm?

9. LEFCOVICH, M. (2009). Kaizen - Detección, prevención y eliminación de desperdicios, una estrategia para la reducción de costos. Argentina: Ed. El Cid.

10. MIRANDA, R. (2005). Competencias claves para la gestión empresarial de las MYPES. Gestión en el tercer milenio. Revista de Investigación de la Facultad de Ciencias Administrativas. UNMSM, Vol. $8\left(\mathrm{~N}^{\circ} 16\right)$.

11. Organización Internacional de Estandarización - ISO . (2008). Norma Internacional ISO 9001:2008. Sistemas de Gestión de la Calidad.

12. PÉREZ, R. (Enero-Abril de 2009). Análisis empírico de la aplicación del modelo de modernización de la gestión para organizaciones en PYMES colombianas. Revista de la Escuela de Administración de Negocios $\left(\mathrm{N}^{\circ} 65\right)$.

13. Perú. Ministerio de la Producción. (2011). CODEMYPE Estadísticas. Lima.

14. Perú. Ministerio de la Producción. (2011).Estadísticas. Dirección General de MYPE y Cooperativas.

15. Perú. Ministerio de la Producción. (2010). Estadísticas de la micro y pequeña empresa 2010. . Dirección General de MYPE y Cooperativas.

16. Small and Medium Enterprise Agency. (1955). Small Business in Japan. Tokyo.

17. USA. Small Business Administration (SBA). (1994). Declaración de impuestos 1994.

18. VILLARÁN, F. (2000). Las PYME's en la estructura empresarial peruana. SASE - Perú. 